\title{
Using the health belief model to identify barriers to seasonal influenza vaccination among Australian adults in 2019
}

\author{
Mallory Trent ${ }^{1}$, Daniel Salmon ${ }^{2}$, and C. Raina MacIntyre ${ }^{1}$ \\ ${ }^{1}$ University of New South Wales \\ ${ }^{2}$ Johns Hopkins University Bloomberg School of Public Health
}

December 20, 2020

\begin{abstract}
Background: Tens of thousands of Australians become ill with influenza annually, causing thousands of severe infections that require hospitalisation. However, only $40 \%$ of adults receive the annual influenza vaccine. We surveyed Australian adults to provide up-to-date data on the predictors and barriers of seasonal influenza vaccination. Methods: We administered an online survey to a nationally representative sample of Australian adults. We designed survey questions using the constructs of the health belief model. Using simple and multivariable Poisson regression, we identified attitudes and beliefs associated with influenza vaccination in 2019. Results: Among 1,444 respondents, 51.7\% self-reported influenza vaccination in 2019. We estimated vaccine coverage to be $44 \%$ for adults under $45,46 \%$ for adults aged 45 to 64 , and $77 \%$ for adults aged 65 and over. The strongest individual predictors of self-reported vaccination were believing the vaccine is effective at preventing influenza $(\mathrm{APR}=3.71 ; 95 \% \mathrm{CI}=2.87-4.80)$, followed by recalling their doctor recommending the vaccine $(\mathrm{APR}=2.70 ; 95 \% \mathrm{CI}=$ 2.31-3.16). Common perceived barriers that predicted self-reported vaccination included believing the vaccine could give you influenza $(\mathrm{APR}=0.59 ; 95 \% \mathrm{CI}=0.52-0.67)$, believing the vaccine can make you ill afterwards $(\mathrm{APR}=0.68 ; 95 \% \mathrm{CI}=$ $0.62-0.74)$, and preferring to develop immunity "naturally" ( $\mathrm{APR}=0.38 ; 95 \% \mathrm{CI}=0.32-0.45)$. Conclusion: Although vaccine uptake in 2019 appears to be higher than previous years, there are perceived barriers which may limit uptake among Australians. Tailored interventions are needed to combat widespread influenza vaccine hesitancy, particularly among high risk grou
\end{abstract}

\section{Hosted file}

Trent et al. Manuscript.pdf available at https://authorea.com/users/383914/articles/499737using-the-health-belief-model-to-identify-barriers-to-seasonal-influenza-vaccinationamong-australian-adults-in-2019 